To appear in Phys. Rev. E 55 (1997)

nucl-ex/9612007

INFNCA-TH9613

\title{
Search for non-Poissonian behavior in nuclear $\beta$ decay
}

\author{
Giorgio Concas ${ }^{1,2, \text { 日 }}$ and Marcello Lissia ${ }^{3,1, \dagger}$ \\ ${ }^{1}$ Dipartimento di Scienze Fisiche, Università di Cagliari, via Ospedale 72, I-09124 Cagliari, Italy \\ ${ }^{2}$ Istituto Nazionale per la Fisica della Materia, via Ospedale 72, I-09124 Cagliari, Italy \\ ${ }^{3}$ Istituto Nazionale di Fisica Nucleare, Sezione di Cagliari, via Negri 18, I-09127 Cagliari, Italy
}

(November 1996)

\begin{abstract}
We performed two independent counting experiments on a $\beta$-emitting source of ${ }_{62}^{151} \mathrm{Sm}$ by measuring the $\gamma$ photon emitted in a fraction of the decays. For counting times ranging from $10^{-3}$ to $5.12 \times 10^{4}$ seconds, our measurements show no evidence of deviations from Poissonian behavior and, in particular, no sign of $1 / f$ noise. These measurements put strong limits on non-Poissonian components of the fluctuations for the subset of decays accompanied by $\gamma$, and corresponding limits for the total number of $\beta$ decays. In particular, the magnitude of a hypothetical flicker floor is strongly bounded also for the $\beta$ decay. This result further constrains theories predicting anomalous fluctuations in nuclear decays.
\end{abstract}

PACS numbers: 05.40.+j, 02.50.-r, 23.90.+w 


\section{INTRODUCTION}

The statistics of the radioactive decay of heavy nuclei have been the subject of much experimental and theoretical work in the past decade. So wide an interest was stimulated by the conjecture that, owing to the intrinsic fluctuations of the decay rate, the counting statistics could depart from the simple Poissonian behavior [1 1 ].

The experimental results are often conflicting, even for the same kind of source. On the one hand, there exist investigations both on $\alpha\left({ }_{95}^{241} \mathrm{Am}\right.$ [ 8 [1] and ${ }_{84}^{210} \mathrm{Po}$ [12]) and $\beta$ decays $\left({ }_{55}^{137} \mathrm{Cs}[13]\right)$ that confirm the Poissonian nature of these processes. On the other hand, several experiments carried out both with $\alpha\left({ }_{95}^{241} \mathrm{Am},{ }_{94}^{239} \mathrm{Pu}\right.$, and ${ }_{96}^{244} \mathrm{Cm}$ [14 [17]) and with $\beta$ sources $\left({ }_{81}^{204} \mathrm{Tl}\right.$ [18], ${ }_{39}^{90} \mathrm{Y}$ [19] and ${ }_{38}^{90} \mathrm{Sr}_{-}^{90} \mathrm{Y}$ [20]), find that the counting variance, for long counting periods, is higher than the Poissonian value by more than one order of magnitude.

This anomalous large variance has been taken as an experimental evidence that the power spectrum of the decay-rate fluctuations has a contribution that grows as the inverse of the frequency $f$ at low frequencies, in addition to the usual frequency-independent Poissonian component. Several mechanisms have been proposed as possible sources of this $1 / f$ noise: quantum self-interference between the wave packets of the emitted particles [3, [1, solidangle fluctuations and random rearrangements within the source [6], spatial $1 / f$ noise in the detector [17]. As a matter of fact, the interpretation of the decay experiments reporting a variance in excess of the Poisson value is still an open problem [6].

In previous work [21,22] we considered the decay statistics of a $\gamma$ source $\left({ }_{50}^{119 m} \mathrm{Sn}\right)$. In that case, we measured that, for counting periods $T$ longer than one hour, the variance of the decay rate significantly deviated from the Poissonian prediction. However, that behaviour could be fully explained by taking into account the time dependence of the statistics 23 without resorting to any exotic effect [21,22].

The aim of this article is to extend our experimental study to a different nucleus, ${ }_{62}^{151} \mathrm{Sm}$, that undergoes $\beta$ decay. There are in fact theoretical claims [6] that deviations from Poissonian statistics could be caused by self-interference of the emitted particles and that these deviations should be present only in $\beta$ decays and not in $\gamma$ or $\alpha$ decays.

\section{EXPERIMENTAL SETUP}

The mean lifetime of ${ }_{62}^{151} \mathrm{Sm}$ is $(130 \pm 12)$ years [24]. While most of the nuclei directly $\beta$ decay into the ground state of ${ }_{63}^{151} \mathrm{Eu}$, a small fraction $(0.91 \%) \beta$-decays into an excited state of energy $E_{\text {exc }}=(21.532 \pm 0.068) \mathrm{keV}$, which then decays to the ground state (mean lifetime: $1.38 \times 10^{-8}$ seconds). In the $3.45 \%$ of cases, this second fast transition produces a $21.532 \mathrm{keV} \gamma$-photon: our apparatus has been set up to detect this photon. In summary, our measurement has the characteristic of selecting a fraction $\xi$ of the total decays $(\xi=$ $\left.(3.14 \pm 0.22) \times 10^{-4}\right)$ : those decays that go through the two-step process, $\beta$ emission followed by a $21.532 \mathrm{keV}$ photon [24]. We shall discuss later and in the Appendix why and to what extent our results on the statistics of the $\gamma$ 's also carry information on the total statistics of the $\beta$ decays.

The source is a crystal of $\mathrm{SmF}_{3}$ containing ${ }_{62}^{151} \mathrm{Sm}$ nuclei (activity: $3.7 \mathrm{GBq}$ ) shaped as a thin disk (diameter: $14 \mathrm{~mm}$ ) with an aluminium cap. The aluminium cap, which closes 
the source, filters out the $\beta$ particles. In the two experiments, which we denote $\mathrm{A}$ and $\mathrm{B}$, we used the same source at different distances (about 15 and $7 \mathrm{~cm}$, respectively) from the detector in order to change the count rate: while Poissonian statistics only depends on the total number of counts (rate $\times$ time), deviations from the standard case (and/or systematic errors) could in principle depend also on the rate (see Ref. 21] for one such an example) and it is better to have the possibility of performing this kind of checks.

In each experiment the photons were detected by a disk-shaped crystal of $\mathrm{NaI}(\mathrm{Tl})$ (diameter: $5 \mathrm{~cm}$ ) integrally mounted on a photomultiplier tube (PMT): we used a crystal 1 $\mathrm{mm}$ thick in experiment $\mathrm{A}$, and a crystal $2 \mathrm{~mm}$ thick in experiment $\mathrm{B}$.

In both experiments, the output signal from the PMT, after being amplified and shaped to a Gaussian pulse, passed through a single-channel analyzer, which selected pulses corresponding to an energy window from 2 to $53 \mathrm{keV}$. The pulse-shaping time constant was $0.5 \mu \mathrm{s}$ and the time resolution of the single-channel analyzer $0.6 \mu \mathrm{s}$. The dead time of the entire system was about $2.5 \mu \mathrm{s}$. The energy window was preliminarily set by means of a multi-channel analyzer module; we have verified that no appreciable drift of the window occurred during the experiments, which lasted 76 days (A) and 19 days (B). We verified that the stability of the energy window and of the voltage of the power supply were sufficient to keep systematic variations of the counting rate below $0.01 \%$, therefore below the statistical fluctuations we measured: only for the longest measurements (total counts of the order of $\left.10^{8}\right)$ the fluctuations-to-signal ratio was as low as $10^{-4}\left(1 / \sqrt{10^{8}}\right)$.

Counting was executed by a programmable multi-channel scaler (MCS) module interfaced to an IBM PC, which provides for control and data storage. In experiment A (B), a set of 40 (38) values of the counting period $T$ was preliminarily defined in the control program with $T$ ranging from $T_{\min }=T_{1}=10^{-3}$ s to $T_{\max }=T_{40}=2^{9} \times 100 \mathrm{~s}\left(T_{\max }=T_{38}=2^{7} \times 100 \mathrm{~s}\right)$. For each value of $T$ the MCS module counted the events occurring in each of 64 consecutive periods of length $T$. Count data were saved on hard disk for further off-line analysis. At the end of experiment A (B), data were available as 40 (38) sequences of 64 counts $M_{k}^{T}$ $(k=1, \ldots, 64)$, one for each of the prefixed values of $T$.

\section{RESULTS AND DISCUSSION}

We analyzed the data by computing the average count and the Allan variance (see Refs. [5.25] and references therein) as function of the time interval $T$. All our results originate from a single uninterrupted run (for each experiment), have been averaged over the same number (64) of consecutive intervals, and are statistically independent (each count has been used only once).

First we verified that the count rate during each experiment had no drifts that could bias the Allan variance; in particular, the slow exponential decay of the source could not affect the Allan variance at the low count rates we operated [21]. Therefore, it is consistent that we consider a constant average rate. We measured this average rate

$$
m=\sum_{T} \frac{1}{T} \frac{1}{64} \sum_{k=1}^{64} M_{k}^{T}
$$


finding $m=(5.3687 \pm 0.00025) \times 10^{3} \mathrm{count} / \mathrm{s}$ in experiment $\mathrm{A}$ and $m=(2.4262 \pm 0.00022) \times$ $10^{4}$ count/s in experiment B. Being the rate constant, the average count for an interval of length $T$

$$
\bar{M}(T) \equiv \frac{1}{64} \sum_{k=1}^{64} M_{k}^{T}
$$

has an expectation value proportional to $T:\langle\bar{M}(T)\rangle=m T$.

There are two reasons for using the Allan variance, which we estimate with an average over 63 consecutive measurements

$$
A(T) \equiv \frac{1}{2 \times 63} \sum_{k=1}^{63}\left[M_{k}^{T}-M_{k+1}^{T}\right]^{2},
$$

instead of the usual variance. The first and most important reason is that $A(T)$ is finite even when the power spectrum grows as $1 / f$ at low frequencies: when non-Poissonian fluctuations might be present, the Allan variance is then a common choice. We remind that the power spectrum of Poissonian fluctuations is independent of frequency: $S(f)=2 m T$; since counts are uncorrelated, the Allan and the usual variance have the same expectation (use $\left\langle M_{j}^{T} M_{k}^{T}\right\rangle \propto \delta_{j k}$ and Eq. (函)), namely, the average count: $\langle A(T)\rangle=\langle\bar{M}(T)\rangle=m T$. However, if the fluctuations have a power spectrum $S(f)=C / f(C$ is a constant independent of $f)$, i.e., we are in presence of $1 / f$ noise, the expectation value of $A$ is: $\langle A(T)\rangle=C \ln 4 T^{2}$ (note the different power of $T$ compared to Poissonian fluctuations), while the usual variance is infinite [5]. A second additional advantage of using the Allan variance is that it is less sensible to drifts of the count rate: the correction is independent of the number of intervals (64) and not proportional to it, see the Appendix of Ref. [21].

Before discussing our results, we wish to comment on our choice of observing the channel of the decay characterized by the emission of a $21.532 \mathrm{keV}$ photon. A more detailed discussion can be found in the Appendix. We made this choice because we can control better the stability of our measurements when detecting photons than when detecting electrons, given our present equipment. However, since one of the motivations of our experiment was to study fluctuations in a $\beta$ decay, it is natural to ask to what extent the statistics of the $\gamma$ emission reflects the statistics of the $\beta$ decay. The time delay of the emission is so small (mean lifetime of the excited state: $1.38 \times 10^{-8}$ seconds) compared to the time intervals of interest that its effect is negligible. Yet one might worry that the fluctuations of the small branching ratio (the fraction of decays that on average emit the $\gamma$ is only $\xi=0.000314$ ) might overwhelm any exotic effect of the original decay. The explicit calculation reported in the Appendix shows that: (1) an upper bound on the flicker floor in the statistics of the $\gamma$ 's implies an equal bound on the flicker floor in the statistics of the parent decay; (2) an upper bound on the ratio of $1 / f$ noise to Poissonian noise in the statistics of the $\gamma$ 's implies a corresponding bound for the statistics of the parent decay weaker by a factor $1 / \xi$; (3) upper bounds on less singular, e.g. frequency independent, deviations from Poissonian behavior in the statistics of the $\gamma$ 's imply corresponding bounds on the parent decay: these bounds on the statistics of the $\beta$ decay are also weaker by a factor $1 / \xi \approx 3000$.

We report in Figs. 11 and 2 the ratio $R(T) \equiv A(T) / M^{2}(T)$ (reduced Allan variance) versus the inverse of the number of counts $1 / M(T)$ for experiments $\mathrm{A}$ and $\mathrm{B}$, respectively. 
Both experiments show that $R(T)$ depends linearly on $1 / M(T)=1 /(m T)$ with unit slope in the range of $T$ considered.

The data perfectly fit the Poisson prediction $R(T)=M / M^{2}=1 / M \propto 1 / T$; this prediction is also reported in Figs. 1 and 2 as a solid line. A fit to the data yields $M(T) \times R(T)=0.99 \pm 0.02$.

On the contrary, a power spectrum $S(f)=C / f$ would yield $R(T)=\left(C \ln 4 T^{2}\right) /(m T)^{2}=$ $C \ln 4 / m^{2}$. Therefore, if we suppose that both a Poissonian and a $1 / f$ contribution are present, when $T$ is large enough the Poissonian contribution becomes negligible and $R(T)$ goes to a constant $\left(F \equiv C \ln 4 / m^{2}\right)$ : this constant $F$ is usually called flicker floor. We measured values of $R(T)$ as low as $6 \times 10^{-9}\left(3 \times 10^{-9}\right)$ in the experiment $\mathrm{A}(\mathrm{B})$ at $T_{\max }=$ $5.12 \times 10^{4} \mathrm{~s}\left(T_{\max }=1.28 \times 10^{4} \mathrm{~s}\right)$ without seeing deviations from Poissonian behavior and, in particular, no signal of the curve turning up at large $T$ and becoming constant. Therefore, we conclude that, if a flicker floor is present, $F<3 \times 10^{-9}$; as discussed in the Appendix, this limit is valid also for the $\beta$ decay.

If we express the power spectrum as the sum of the Poissonian component plus a hypothetical $1 / f$ component: $S(f)=2 m T+C / f$, in the range of frequencies accessible by our experiments $\left(f>1 / T_{\max }\right)$, the limit on the flicker floor implies an upper limit on the ratio of the strength of the $1 / f$ contribution $(C / f)$ relative to the Poissonian one $(2 m T)$, i.e., a limit on the ratio $(C / f) /\left(2 m T_{\max }\right)<C /(2 m):(C / f) /\left(m T_{\max }\right)<1 \times 10^{-5}$ $\left(C / m<2.5 \times 10^{-5}\right)$. These limits on the strength of the $1 / f$ noise are valid for the channel of the decay with $\gamma$ emission; for the total $\beta$ decays the limit is weaker (see Appendix): $\left(C_{\beta} / f\right) /\left(m_{\beta} T_{\max }\right)<3 \times 10^{-2}$.

The model of quantum $1 / f$ noise proposed by Handel predicts $F=8 \alpha \zeta \ln 2(\Delta v / c)^{2} /(3 \pi)$ for $\beta$ decays, see Eq. (3.6) of Ref. [5], and references therein; here $\alpha \approx 1 / 137$ is the fine structure constant, $0<\zeta<1$ is a coherence factor, and $\Delta v / c$ is the velocity change of the particles in the emission process relative to the speed of light $c$ : if $K_{\beta}$ is the kinetic energy of the electron, $(\Delta v / c)^{2}=1-\left[1+K_{\beta} /\left(m c^{2}\right)\right]^{-2}$. Since we did not measured the electron energy, our data are averaged over the entire electron-energy spectrum. Therefore, we can only give an estimate of the limit on the coherence factor by using the average electron energy: $\left\langle K_{\beta}\right\rangle=13.96 \mathrm{keV}$. The fact that we do not see any flicker floor implies, in the context of Handel's model, that the coherence factor $\zeta$ must be smaller than about $10^{-5}$. Our limit should be compared to the recent positive determinations of $\zeta$ in the range $5.2 \times 10^{-3}<\zeta<8.3 \times 10^{-3}$, that Gopala et al. [20] have made, albeit in different $\beta$ decays: ${ }_{38}^{90} \mathrm{Sr},{ }_{39}^{90} \mathrm{Y}$ and ${ }_{81}^{204} \mathrm{Tl}$. We do not have any explanation why $\zeta$ should be more than two orders of magnitude larger in those decays compared to our upper limit.

\section{CONCLUSIONS}

We have measured the counting rate of secondary $\gamma$ rays from a $\beta$ source of ${ }_{62}^{151} \mathrm{Sm}$ for counting periods ranging from $10^{-3}$ to $5.12 \times 10^{4}$ seconds, and studied the fluctuations of the rate by means of the Allan variance.

- We have found no evidence of deviations from Poissonian behavior up to a ratio of fluctuations to signal as low as $5 \times 10^{-5}$. 
- The ratio between a hypothetical $1 / f$ component of the power spectrum and the usual Poissonian contribution must be less than $1 \times 10^{-5}$ at the longest time interval (lowest frequency) that we have measured $\left(T_{\max }=5.12 \times 10^{4} \mathrm{~s}\right)$.

- We found no evidence of flicker floor. The upper bound on a hypothetical flicker floor is $3 \times 10^{-9}$; this limit is valid also for the statistics of the total $\beta$ decays.

- If our upper bound the flicker floor is interpreted in the context of Handel's theory of $1 / f$ noise predicting coherent interference of the emitted charged particle, the coherence factor $\zeta$ for this decay must be less than about $1 \times 10^{-5}$ : this number is more than two orders of magnitude smaller than the one that has been recently proposed in the literature albeit for different decays [20].

\section{ACKNOWLEDGMENTS}

We gratefully acknowledge stimulating and encouraging discussions with R. Boscaino.

This work has been partially supported by M.U.R.S.T. (Italian Ministry of University and Scientific and Technological Research).

\section{APPENDIX:}

In this Appendix, we discuss the relation between fluctuations of the number of total decays (in our case, the total number of $\beta$ decays) and fluctuations of the number of decays in a subchannel (in our case, the fraction of $\beta$ decays that produce a $\gamma$ photon with energy $21.532 \mathrm{keV})$.

The main results of this Appendix are summarized by Eqs. (A8) and (A9).

We consider only the effect of the fluctuations of the branching ratio and not the effect of time delay between the first and second decay, which in general has the effect of a low-pass filter [9], since this second decay (the $\gamma$ emission) is practically instantaneous for the case under study (mean lifetime $13.8 \mathrm{~ns}$ compared to a time resolution of the order of $\mu$ s and to the shortest time interval considered: $1 \mathrm{~ms}$ ).

In the following we shall use the symbol $M$ when referring to the number of detected $\gamma$ 's and the symbol $N$ when referring to the corresponding (total) number of $\beta$ decays. We shall also use the subscript $\gamma(\beta)$ referring to the partial daughter statistics (total parent statistics). Let us define two kinds of averages:

$\langle\cdots\rangle_{N}$ average over the $\gamma$-count distribution keeping the number of $\beta$ counts $N$ fixed;

$\langle\cdots\rangle$ average over the $\beta$-count distribution.

Using the symbol $\xi$ for the branching ratio, we can write

$$
\begin{aligned}
\langle M\rangle_{N} & =\xi N \\
\bar{M} \equiv\left\langle\langle M\rangle_{N}\right\rangle & =\xi \bar{N}
\end{aligned},
$$

where for simplicity we use the symbol $\bar{M}$ to indicate the number of $\gamma$ counts twice averaged both over the $\gamma$ and $\beta$ distributions and, at the same time, the symbol $\bar{N}$ to indicate the average $\beta$ counts (over the $\beta$ distribution). 
Since our experiments do not show any deviation from Poissonian behavior, we can readily put limits on non-Poissonian components of the $\gamma$ counts. The implications for the total $\beta$ decay can be assessed by assuming that the $\gamma$ distribution at fixed number of $\beta$ decays $N$ is standard (binomial and frequency independent) and by considering the effect of fluctuations of $N$ :

$$
\left\langle\left(M_{i}-\langle M\rangle_{N_{i}}\right)\left(M_{j}-\langle M\rangle_{N_{j}}\right)\right\rangle_{N_{i} N_{j}}=\delta_{i j}\langle M\rangle_{N_{i}}=\delta_{i j} \xi(1-\xi) N_{i}
$$

where the indices $i$ and $j$ indicate different counting intervals.

We first consider the average at fixed $N_{i}$ and $N_{i+1}$ of $\left(M_{i}-M_{i+1}\right)^{2}$, which by adding and subtracting $\langle M\rangle_{N_{i}}=\xi N_{i}$ can be written as

$$
\begin{aligned}
\left\langle\left(M_{i}-M_{i+1}\right)^{2}\right\rangle_{N_{i} N_{i+1}}=\langle & {\left.\left[\left(M_{i}-\langle M\rangle_{N_{i}}\right)-\left(M_{i+1}-\langle M\rangle_{N_{i+1}}\right)+\xi\left(N_{i+1}-N_{i}\right)\right]^{2}\right\rangle_{N_{i} N_{i+1}}(\mathrm{~A}) } \\
= & \left\{\left\langle\left(M_{i}-\langle M\rangle_{N_{i}}\right)^{2}\right\rangle_{N_{i}}+\left\langle\left(M_{i+1}-\langle M\rangle_{N_{i+1}}\right)^{2}\right\rangle_{N_{i+1}}+\xi^{2}\left(N_{i}-N_{i+1}\right)^{2}\right. \\
& -2\left\langle\left(M_{i}-\langle M\rangle_{N_{i}}\right)\left(M_{i+1}-\langle M\rangle_{N_{i+1}}\right)\right\rangle_{N_{i} N_{i+1}} \\
& \left.+2 \xi\left(N_{i}-N_{i+1}\right)\left[\left\langle\left(M_{i}-\langle M\rangle_{N_{i}}\right)\right\rangle_{N_{i}}+\left\langle\left(M_{i+1}-\langle M\rangle_{N_{i+1}}\right)\right\rangle_{N_{i+1}}\right]\right\} \\
= & \xi(1-\xi)\left(N_{i}+N_{i+1}\right)+\xi^{2}\left(N_{i}-N_{i+1}\right)^{2},
\end{aligned}
$$

where we have applied Eq. (A3) to the first and second line of Eq. (A5), while the third line is identically zero.

If we now divide the above result by two and average it over the $\beta$ distribution, we find (considering that for a stationary process $\left\langle N_{i}\right\rangle=\bar{N}$ independently of $i$ ):

$$
\frac{1}{2}\left\langle\left\langle\left(M_{i}-M_{i+1}\right)^{2}\right\rangle_{N_{i} N_{i+1}}\right\rangle=\xi(1-\xi) \bar{N}+\xi^{2} \frac{1}{2}\left\langle\left(N_{i}-N_{i+1}\right)^{2}\right\rangle .
$$

The left-hand side of Eq. (A7) is the expectation value of the Allan variance of the $\gamma$ counts, which we measure with the statistics defined in Eq. (3), while the right-hand side is the expectation value of the Allan variance of the total $\beta$-decay counts. If we define $A_{\gamma}\left(A_{\beta}\right)$ as the Allan variance and $R_{\gamma} \equiv A_{\gamma} / \bar{M}^{2}\left(R_{\beta} \equiv A_{\beta} / \bar{N}^{2}\right)$ as the relative Allan variance of the $\gamma$ $(\beta)$ counts, Eq. (A7) becomes

$$
\begin{aligned}
& A_{\gamma}=(1-\xi) \bar{M}+\xi^{2} A_{\beta} \\
& R_{\gamma}=\frac{(1-\xi)}{\bar{M}}+R_{\beta},
\end{aligned}
$$

which constitute the main result of this Appendix.

In the following we analyzes the consequences of Eq. (A9) for our experimental study.

\section{a. Flicker floor}

If the $\beta$ decay has a $1 / f$ component that produces a flicker floor $F_{\beta}$ in the relative Allan variance, i.e., $R_{\beta}=1 / \bar{N}+F_{\beta}$, the fact that no deviation of $R_{\gamma}$ from $1 / \bar{M}$ has been observed for $R_{\gamma}$ as low as $3 \times 10^{-9}$ implies not only that $F_{\gamma}<3 \times 10^{-9}$, but also that $F_{\beta}<3 \times 10^{-9}$, where we have used Eq. (A9) dropping $\xi \approx 3 \times 10^{-4}$ compared to 1 : $(1-\xi) \approx 1$ and $1 / \bar{M}+1 / \bar{N} \approx 1 / \bar{M}$. 


\section{b. $1 / f$ noise}

If we are interested in the ratio of the $1 / f$ contribution $\left(C_{\beta} / f\right)$ relative to the Poissonian one $\left(2 m_{\beta} T\right)$, we should recall that the rate of the $\beta$ decay is $m_{\beta}=m_{\gamma} / \xi$ and the that the constant $C$ is related to the flicker floor by $C=F \times m^{2} / \ln 4$. Then this ratio for the $\beta$ decay can be related to the same ratio for the $\gamma$ decay by using the fact that the limit on $F_{\beta}$ and the one on $F_{\gamma}$ are equal: $\left(C_{\beta} / f\right) /\left(2 m_{\beta} T_{\max }\right)=\left(F_{\beta} m_{\beta} / f\right) /\left(T_{\max } \ln 4\right)=$ $(1 / \xi) \times\left(F_{\gamma} m_{\gamma} / f\right) /\left(T_{\max } \ln 4\right)$. We loose a factor $1 / \xi \approx 3000$ going from the upper bound on the ratio of the $1 / f$ contribution relative to the Poissonian one for the $\gamma$ statistics $\left(1 \times 10^{-5}\right)$ to the upper bound on the same ratio for the $\beta$ statistics $\left(3 \times 10^{-2}\right)$.

\section{c. Frequency-independent non-Poissonian component}

If instead we suppose that the $\beta$ decay has a frequency-independent deviation from Poissonian statistics, i.e., $R_{\beta}=\kappa / \bar{N}=\kappa \xi / \bar{M}$, the fact that no deviation of $R_{\gamma}=(1-\xi+$ $\kappa \xi) / \bar{M}$ from $1 / \bar{M}$ has been observed $\left(\bar{M} \times R_{\gamma}=0.99 \pm 0.02\right)$ implies also that $(1-\kappa) \xi=$ $0.01 \pm 0.02$ and, consequently, that $\kappa=-30 \pm 60$.

In conclusion, we have show in this Appendix that a measurement of a process ( $\beta$ decay) by selecting a subprocess (detecting the $\gamma$ emitted in a fraction of the decays) whose branching ratio $\xi$ is itself a statistical variable corresponds, as might have been expected, to the use of a detector with efficiency not greater than $\xi$. Therefore, we loose a factor $1 / \xi$ in most limits on dimensionless quantities when passing from statistics of the subprocess to total statistics of entire process. However, there exist quantities, such as the flicker floor, that can be determined from the partial statistics without loosing any sensibility. The reason of this different behavior is related on how strongly the noise under study depends on the number of events $N$ compared to the usual $\sqrt{N}$ dependence. 


\section{REFERENCES}

* Electronic address: concas@alfis.unica.it

$\dagger \quad$ Electronic address: lissia@cagliari.infn.it

[1] P. H. Handel, Phys. Rev. Lett. 34, 1492 (1975). Title: $1 / f$ Noise - An "Infrared Phenomenon".

[2] P. H. Handel, Phys. Rev. Lett. 34, 1495 (1975). Title: Nature of $1 / f$ Noise .

[3] P. H. Handel, Phys. Rev. A 22, 745 (1980). Title: Quantum approach to $1 / f$ noise.

[4] C. M. V. Vliet, P. H. Handel, and A. V. der Ziel, Physica A 108, 511 (1981). Title: Superstatistical emission noise.

[5] C. M. V. Vliet and P. H. Handel, Physica A 113, 261 (1982).

Title: A new transform theorem for stochastic processes with special application to counting statistics,

[6] C. M. V. Vliet, Solid State Electronics 34, 1 (1991).

Title: A survey of results and future prospects on quantum $1 / f$ noise and $1 / f$ noise in general.

[7] F. N. Hooge, in Noise in Physical Systems and 1/f Noise, edited by V. Bareikis and R. Katilius (World Scientific, Singapore, 1995), p. 8., and other articles in the book. Title: $1 / f$ noise in semiconductor materials.

[8] W. V. Prestwich, T. J. Kennett, and G. T. Pepper, Phys. Rev. A 34, 5132 (1986). Title: Search for $1 / f$ fluctuation in $\alpha$ decay.

[9] W. V. Prestwich, T. J. Kennett and G. T. Pepper, Can. J. Phys. 66, 100 (1988). Title: Comment on: Flicker noise fluctuations in $\alpha$-radioactive decay.

[10] G. T. Pepper, T. J. Kennett, and W. V. Prestwich, Can. J. Phys. 67, 468 (1989). Title: A re-investigation of the possibility of $1 / f$ noise fluctuations in $\alpha$ decay.

[11] T. J. Kennett and W. V. Prestwich, Phys. Rev. A 40, 4630 (1989). Title: Limit on the existence of $1 / f$ noise in $\alpha$ decay.

[12] M. A. Azhar and K. Gopala, Phys. Rev. A 39, 5311 (1989). Title: Search for $1 / f$ fluctuations in $\alpha$ decay of ${ }^{210} \mathrm{Po}$.

[13] K. Gopala and M. A. Azhar, Phys. Rev. A 37, 2173 (1988). Title: Search for $1 / f$ fluctuations in $\gamma$ decay of ${ }^{137} \mathrm{Cs}$.

[14] J. Gong, C. M. Van Vliet, W. H. Ellis, G. Bosman and P. H. Handel, in Noise in Physical Systems and $1 / f$ Noise, edited by G. L. M. Savelli and J. P. Nougier (Elsevier, New York, 1983), p. 381.

Title: $1 / f$ noise fluctuations in $\alpha$-particle radioactive decay of ${ }^{241} \mathrm{Am}$.

[15] G. S. Kousik, C. M. Van Vliet, G. Bosman, W. H. Ellis and E. E. Carrol, in Noise in Physical Systems and 1/f Noise, edited by A. D'Amico and P. Mazzetti (Elsevier, New York, 1986), p. 469.

Title: $1 / f$ noise in alpha-particle decay of ${ }^{239} \mathrm{Pu},{ }^{241} \mathrm{Am}$ and ${ }^{244} \mathrm{Cm}$.

[16] G. S. Kousik et al., Can. J. Phys. 65, 365 (1987).

Title: Flicker-noise fluctuations in $\alpha$-radioactive decay.

[17] V. D. Rusov et al., Nucl. Tracks Radiat. Meas. 20, 305 (1992). 
Title: Observation of spatial $1 / f$ noise in experimental detection of ${ }^{239} \mathrm{Pu} \alpha$-particles by solid state nuclear track detector.

[18] M. A. Azhar and K. Gopala, Phys. Rev. A 39, 4137 (1989).

Title: $1 / f$ noise in $\beta^{-}$decay of ${ }^{204} \mathrm{Tl}$.

[19] M. A. Azhar and K. Gopala, Phys. Rev. A 44, 1044 (1991).

Title: $1 / f$ fluctuations in $\beta^{-}$decay of ${ }^{90} Y$.

[20] K. Gopala, M. A. Azhar and Swamy, Phys. Rev. E 50, 2588 (1994).

Title: $1 / f$ noise in the $\beta$ decay of ${ }^{90} \mathrm{Sr}_{-}{ }^{90} \mathrm{Y}$.

[21] R. Boscaino, G. Concas, M. Lissia, and S. Serci, Phys. Rev. E 49333 (1994).

Title: Fluctuations in radioactive decays. I. Nonequilibrium effects and noise.

[22] R. Boscaino, G. Concas, M. Lissia, and S. Serci, Phys. Rev. E 49341 (1994).

Title: Fluctuations in radioactive decays. II. Experimental results.

[23] M. C. Teich and H. C. Card, Opt. Lett. 4, 146 (1979).

Title: Photocounting distribution for exponentially decaying sources.

[24] R. B. Firestone, Table of Isotopes, 8th edition (John Wiley \& Sons, New York, 1996).

[25] W. V. Prestwich, T. J. Kennett, and F. W. Kus, Can. J. Phys. 69, 1405 (1991).

Title: The statistical properties of Allan variance. 


\section{FIGURES}

FIG. 1. Relative Allan variance $R(T)$ versus the inverse mean number of decays $1 / \bar{M}$ (lower scale) and the inverse time interval $1 / T$ (upper scale) for the experiment A (average rate $\left.m \equiv M / T=5.3687 \times 10^{3}\right)$. Diamonds are the experimental values. The solid line is the Poissonian prediction $R=1 / M$.

FIG. 2. Same as Fig. 1 for the experiment B (average rate $m=2.4262 \cdot 10^{4}$ ). 


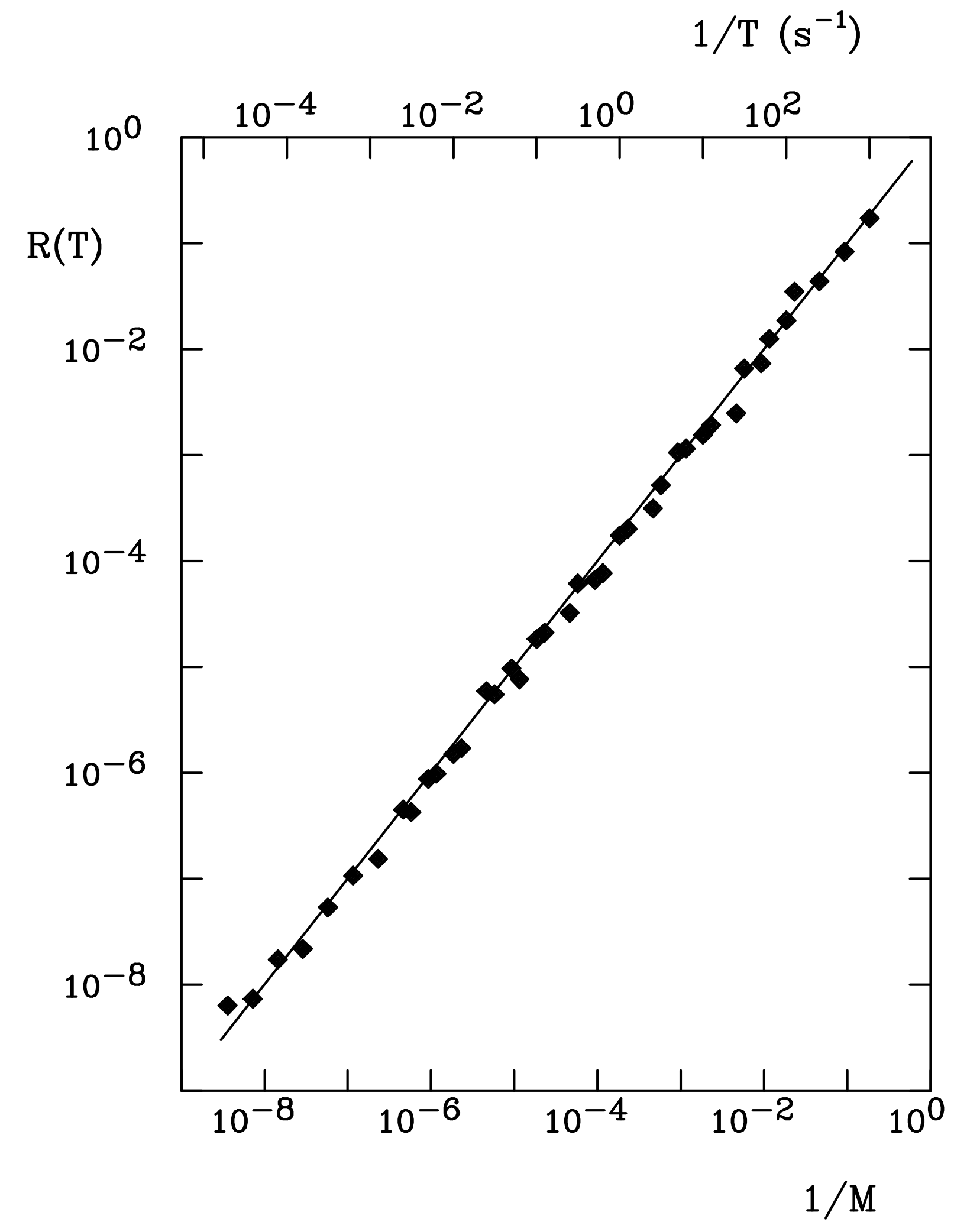

$F_{1 g} 1$ 


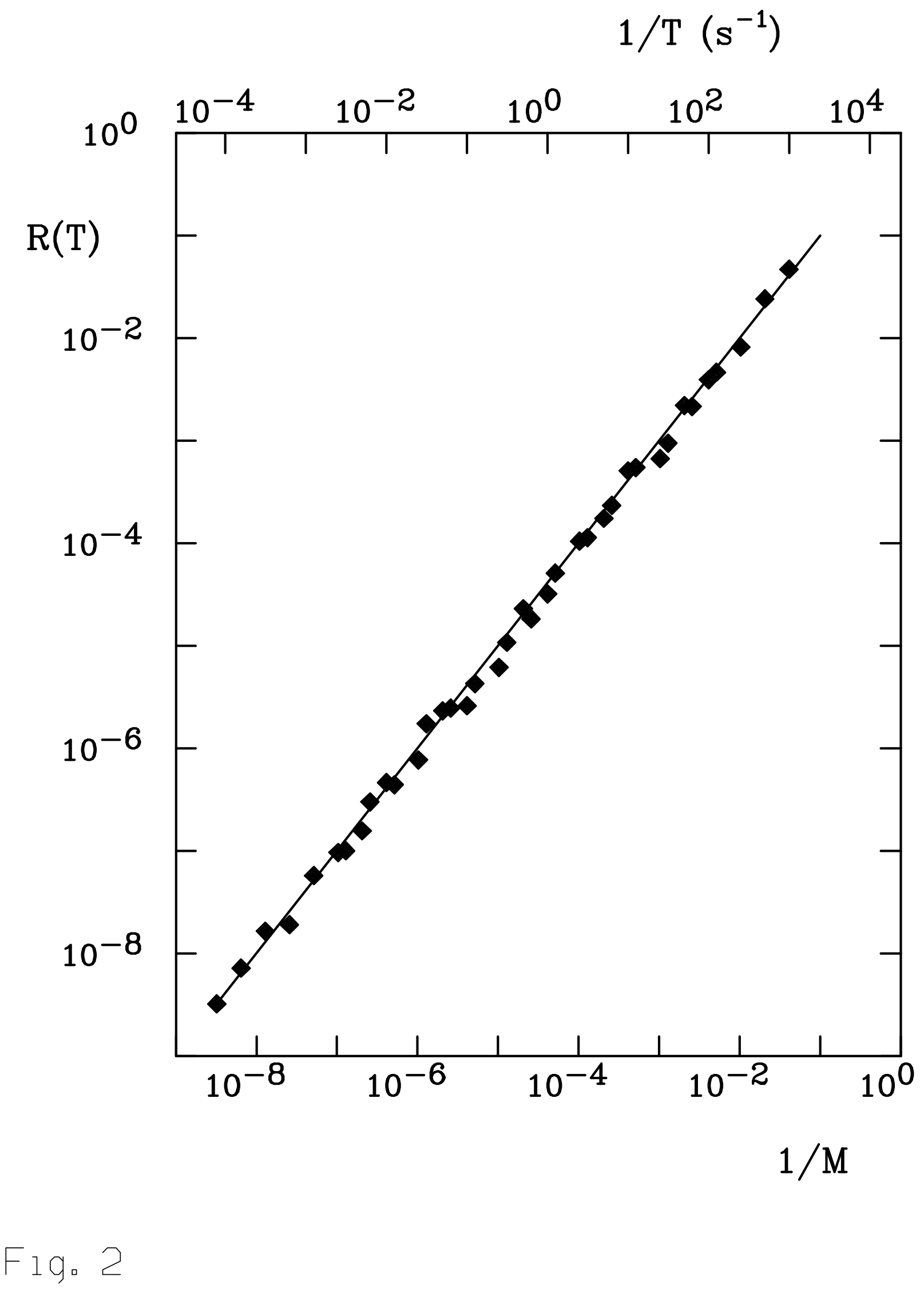

